\title{
Violência e Outras Vulnerabilidades de Gênero em Mulheres Vivendo com HIVIAids
}

\author{
Márcia de Lima ${ }^{1}$ \\ Faculdade de Medicina da Universidade de São Paulo, Programa de Pós-Graduação \\ do Departamento de Medicina Preventiva, São Paulo, Brasil \\ Lilia Blima Schraiber \\ Faculdade de Medicina da Universidade de São Paulo, São Paulo, Brasil
}

\begin{abstract}
Resumo
Na feminização do HIV e na articulação com alguns estudos sobre as desigualdades de gênero, identificamos algumas particulares situações contextuais das mulheres que implicam em grandes dificuldades materiais ou simbólicas para que elas se protejam da infecção ou consigam desenvolver comportamentos de maior cuidado em saúde. Isto nos leva a propor no presente artigo de reflexão teórica a 'vulnerabilidade de gênero' como categoria norteadora para estudos sobre contextos de vida de mulheres em que os componentes afetivos e as situações de violência contribuam para as dificuldades, sejam do cuidado na sua saúde, quando portadoras de HIV, ou na prevenção da doença. Considera-se, portanto, a feminizaçao da aids e as relações desta com a vulnerabilidade de gênero a que as mulheres estão submetidas, com destaque às suas situações de violência. Tais situações operam como um contexto desencadeador de novas vulnerabilidades relativamente às trazidas pela soropositividade e evidenciam o quanto a violência e a condição de portadora do HIV se reforçam mutuamente como vulnerabilidades, agravando para essas mulheres as condições de subordinação de gênero.
\end{abstract}

Palavras-chave: Vulnerabilidade, gênero, violência contra a mulher, Síndrome da Imunodeficiência Adquirida.

\section{Violence and Other Gender Vulnerabilities and Women Living with HIVIAids}

\begin{abstract}
In the process of feminization of HIV and in conjunction with some studies on gender inequalities, we identify some particular contextual situations involving women in big trouble material or symbolic ones, that difficult to protect themselves for infection or to take care of their health. The propose of this article is to present a theoretical discussion about 'vulnerability gender' as a category for guiding studies on women's life contexts in which the affective components and situations of violence increase difficulties in their health care, when HIV-infected, or in the prevention of disease. Considered, therefore, the aids feminization condition and its relations to gender vulnerability that women are subjected, especially to their violences situations. Such situations act as a trigger of context in relation to new vulnerabilities brought about by HIV status and show how violence and HIV-positive status are mutually reinforcing as vulnerabilities, worsening conditions for these women of gender subordination.
\end{abstract}

Keywords: Vulnerability, gender, violence against women, Acquired Immune Deficiency Syndrome.

Endereço para correspondência: Faculdade de Medicina da Universidade de São Paulo, Programa de PósGraduação do Departamento de Medicina Preventiva, Av. Dr. Arnaldo, 455, $2^{\circ}$ andar, sala 2170, Cerqueira César, São Paulo, SP, CEP 01246-903.E-mail: marcia-li@uol.com.br e liliabli@usp.br 


\section{Violencia y Otras Vulnerabilidades de Género en las Mujeres Que Viven com el HIV/Sida}

\section{Resumen}

En feminización del VIH y la articulación con algunos estudios en torno a las desigualdades de género, se han identificado algunas situaciones contextuales específicas de las mujeres que implicar grandes dificultades materiales o simbólica a ellos para protegerse de la infección o desarrollar conductas de mayor atención de la salud. Esto nos lleva a proponer en este artículo de la Reflexión Teórica la "vulnerabilidad de género" como categoria de orientar los estudios sobre contextos de la vida de la mujer en la que los componentes afectivos y situaciones de violencia contribuyen a las dificultades, están en su atención médica, cuando se vive con el VIH o en la prevención de la enfermedad. Es, por lo tanto, la feminización del SIDA y su relación con la vulnerabilidad del género que son objeto las mujeres, especialmente a su violencia. Este tipo de situaciones funcionan como un contexto de activación de las vulnerabilidades relativamente nuevos traídos por la seropositividad y mostrar cómo el estado de violencia y VIH-positivos se refuerzan mutuamente como vulnerabilidades, lo que agrava las condiciones de estas mujeres la subordinación de género.

Palabras clave: Vulnerabilidad, el género, la violencia contra la mujer, el Síndrome de Inmunodeficiencia Adquirida.

\section{A Questão da Feminização da Epidemia HIV/Aids}

Apesar do desenvolvimento progressivo no cuidado às pessoas vivendo com HIV e aids, dos trabalhos preventivos e da disponibilização de insumos, tais como preservativos, a epidemia da aids no Brasil e no mundo ainda é um importante problema de saúde pública e, tornar-se uma condição de vida similar às doenças crônicas, coloca novas e preocupantes questões para seu controle. Uma delas é a feminização crescente desde os anos 1990, que é hoje um dos desafios no enfretamento da infecção pelo HIV.

Segundo a UNAIDS (Joint United Nations Program on HIV/AIDS, 2008), estima-se que no cenário mundial há 33 milhões de pessoas no mundo vivendo com HIV, dentre elas 15,5 milhões são mulheres acima de 15 anos, o que representa $50 \%$ do total de adultos infectados. O estudo indica que $77 \%$ (12 milhões) de todas as mulheres HIV positivas vivem na África Subsaariana, o que representa $59 \%$ dos adultos vivendo com HIV. Observa-se também que esta prevalência entre mulheres jovens, é três vezes maior do que a prevalência entre homens jovens (15 a 24 anos). Na Ásia as mulheres representam $35 \%$ (1,7 milhão) dos adultos vivendo com HIV. A UNAIDS informa, ainda, que no Caribe 50\%
(110 mil) dos adultos vivendo com HIV são mulheres. No Leste Europeu, há um crescimento no número de mulheres infectadas pelo HIV, sendo a Ucrânia o país caracterizado por uma das epidemias que crescem mais rapidamente ( $44 \%$ dos adultos infectados são mulheres). Na América Latina, uma crescente proporção de pessoas vivendo com HIV são de mulheres (550 mil).

O cenário nacional mantém-se estável desde 2004 e estima-se que 630 mil pessoas vivam hoje com HIV/Aids. Foram notificados 608.230 casos de aids no Brasil de 1980 até junho de 2011. Em 2010 foram notificados 34.218 novos casos, com incidência de 17,9/100.000 habitantes e razão de sexo de 1,7 novos casos em homens para cada caso em mulheres. Ao longo dos últimos 12 anos observa-se uma estabilização da taxa de incidência no Brasil, mas por regiões a taxa diminuiu na Sudeste e aumentou nas demais regiões (Ministério da Saúde, 2011).

Em relação aos homens e mulheres, ainda temos mais casos de aids entre homens, mas a diferença vem diminuindo ao longo dos anos. Proporcionalmente o número de casos de aids entre mulheres em 1989 era de 6 casos de aids no sexo masculino para cada 1 caso no sexo feminino. A Razão de sexos em 2010, chegou a 1,7 caso em homens para cada $1 \mathrm{em}$ mulheres. 
Diante desta realidade e das necessidades de ações e estratégias para articular o enfrentamento da vulnerabilidade em que as mulheres estão envolvidas, a Secretaria Especial de Políticas para as mulheres do Ministério da Saúde, por meio do Departamento de DST/Aids e da área Técnica da Saúde da Mulher, em 2007, apresentam o Plano Integrado de Enfrentamento da Feminização da Epidemia da Aids e outras DST ${ }^{2}$, que intra e intersetorialmente reúne esforços e parcerias para abarcar elementos para o enfrentamento das múltiplas vulnerabilidades em que as mulheres estão suscetíveis à aids e outras DST. Este plano foi revisado em $2009^{3}$ como resultado de debates entre a sociedade civil e governo, para concretizar e nortear estratégias de promoção, prevenção e assistência à saúde, como também alterar e considerar os diferentes contextos que vulnerabilizam as mulheres.

Assim, o plano preocupou-se em considerar os componentes econômicos, socioculturais, raciais e étnicos, relevantes na estrutura das desigualdades sociais, além da violência doméstica e sexual contra mulheres, somado às relações de discriminação e preconceito sobre as vivências da sexualidade feminina. Estas questões devem constar das agendas das práticas do cuidado em saúde, visando reduzir desigualdades e adoecimentos. O plano se estrutura a partir da histórica iniciativa dos movimentos sociais e das instituições governamentais, tendo como marco os direitos sexuais e reprodutivos das mulheres.

Questões da vivência da sexualidade, do conhecimento do corpo, da negociação de medidas preventivas, a existência de relações desiguais entre os homens e mulheres, têm papel relevante no processo da feminização do HIV e aids. Fatores relacionados à baixa escolaridade, à forma de inserção social, como dificuldades de colocação em mercado de trabalho ou mesmo grandes desigualdades na remuneração de seu trabalho, também acrescentam as possibilidades de maior vulnerabilidade das mulheres.

http://bvsms.saude.gov.br/bvs/publicacoes/ plano_feminizacao_final.pdf

3 http://sistemas.aids.gov.br/feminizacao/sites/ default/files/PlanoIntegrado-2009.pdf
Dentre estes fatores, chamamos especial atenção para a violência contra a mulher, expressas nas diferentes formas de práticas de abuso e atos de agressões, como violência doméstica, sexual, moral e patrimonial. Esta questão também compõe o elenco de preocupações contempladas pelo Plano de Enfrentamento da Feminização do HIV/aids. Para melhor se abordar a complexidade representada pelas situações que aumentam a vulnerabilidade das mulheres, pode-se lançar mão da perspectiva de gênero, cuja formulação conceitual em muito amplia a compreensão sobre as desigualdades entre homens e mulheres na sociedade e como este problema está articulado com o aumento da infecção pelo HIV em mulheres.

Neste processo as contribuições originárias dos progressivos estudos acerca da violência contra a mulher, em que se destacam as situações perpetradas pelo parceiro íntimo (Heise, Ellsberg, \& Gottemoeller, 1999). Barros, Schraiber e França (2011), Heise e Garcia-Moreno (2002), e Teitelman, Ratclitte, Dichter e Sullivan (2008), vêm somar outro conjunto importante de questões, reforçando as mencionadas relações desiguais entre homens e mulheres. Estudo sobre o tema da violência de gênero e a vulnerabilidade ao HIV/Aids, vem mostrar que a violência também é uma questão para situações de parceria íntima entre casais de mesmo sexo. Como apontam Wong, Weiss, Ayala e Kiple (2010), entre os 526 homossexuais, $12 \%$ foram agressores e $41 \%$ foram vítimas de violência por parceiro íntimo, sendo as agressões emocionais e físicas (23\%) e o abuso sexual (18\%) os principais tipos encontrados. O estudo comenta, ainda, que o a violência vivenciada pela comunidade de Lésbicas, Gays, Bissexuais, Travestis e Transexuais (LGBT) apresenta similar diferença em termos de local de agressão e o nível de intimidade entre a vítima e agressor que o perfil verificado entre homens e mulheres heterossexuais, pois os desconhecidos perpetram mais a violência relacionada à discriminação, em locais públicos, enquanto que a violência doméstica é situação que ocorre entre os parceiros. Outra importante questão relativa às pessoas com práticas homossexuais é o assédio sexual a que estão expostos, tanto entre parceiros quanto por práticas homo- 
fóbicas, o que os colocam em situação de maior vulnerabilidade ao HIV e aids (Huebner, Rebchook, \& Kegeles, 2004).

Estudado esse conjunto de questões da perspectiva das teorias feministas de gênero, pode-se pensar a violência não apenas como questão de conflito em torno das diferenças entre sujeitos no espaço público ou no privado, mas como questão em que a resolução destes conflitos envolvem reafirmações de poder desigual nas relações, reafirmando os padrões hegemônicos de masculinidade.

Dada a pretensão de se examinar em particular a situação das mulheres, com base no referencial de gênero, buscar-se-á observar de que modo a vulnerabilidade das mulheres é perpassada pela desigualdade de gênero socialmente estabelecida e de que forma as situações de violência revelam e acentuam essas desigualdades, representando uma nova base para o controle da epidemia.

\section{Teorias de Gênero e o Conceito de Vulnerabilidade ao HIV/Aids}

As conexões entre desigualdades de gênero e violência, de um lado, e entre desigualdade de gênero e AIDS, de outro lado, enquanto conexões já bem estudadas pela literatura nacional e internacional, reforçam a necessidade de entrecruzarmos também a ocorrência das violências, como uma das questões de gênero, com a crescente vulnerabilidade das mulheres à infecção pelo HIV ou a outras DST, tendo gênero, portanto, como a base comum a todas elas. Isso implica em uma aproximação da vulnerabilidade das mulheres às DST/HIV como desigualdade de poder de sujeito social, a que vêm se somar outras desigualdades, como as sócio-econômicas, as étnico-raciais ou as conexas ao acesso às instituições de saúde, denominada de vulnerabilidade programática.

$\mathrm{O}$ conceito de gênero tem sido utilizado e debatido desde a década de 1970, tratando das diferenças entre homens e mulheres e enfatizando a posição hierárquica superior dos homens. Scott (1990) uma das mais conceituadas referências da teoria de gênero, aponta, também, que em muitos estudos o termo "mulheres" foi sendo substituído pelo termo "gênero", dando o entendimento de sinônimos. No entanto, o termo "gênero" inclui não só as mulheres.

$O$ conceito de gênero traduz um conjunto de normas, valores, costumes e práticas através de que a diferença biológica entre homens e mulheres é culturalmente significada e diferenciada em termos de valor e poder social. Surge como uma forma de distinguir as diferenças biológicas das desigualdades sócio culturalmente construídas e procura mudar a atenção de um olhar para mulheres e homens como segmentos isolados, para um olhar que se fixa nas relações inter-pessoais e sociais.

Neste sentido, os estudos sobre parentesco e os papéis constituídos nas diferentes sociedades, as relações entre natureza e cultura, as dimensões do essencialismo e da universalização sobre o masculino e o feminino, a questão da opressão das mulheres, tornaram-se produtivos para as discussões do movimento social e para os estudos de gênero (Franchetto, Cavalcanti, \& Heilborn, 1981; Rosaldo \& Atkinson, 1975; Rubin, 1986).

Cabe destacar que da mesma forma com que gênero permite identificar a naturalização ou essencialismo dos papéis sociais em termos do sexo biológico, permite, por outro lado, realizar a desconstrução dessas compreensões. O maior poder masculino frente ao poder das mulheres como sujeitos sociais é uma construção social e histórica, que não deve ser entendido como uma hierarquia do mundo natural. Assim, o uso da perspectiva de gênero permite também rejeitar tal construção e, ao revés, elaborar de sua crítica com a proposição de maior equidade nas relações entre homens e mulheres. As questões de gênero permeiam a história de mulheres e de homens e as relações entre essas histórias, permitindo também notar a multiplicidade de conexões que contemplam não somente as hierarquias entre eles, mas aquelas de classe social, raça e etnia.

Uma questão importante formulada por Firestone (1976) e Franchetto et al. (1981) diz respeito à maternidade como componente do biológico que geraria a opressão feminina. Já para Reed (1973) a opressão feminina estaria mais relacionada ao fato da mulher não se colocar como uma trabalhadora para a produção social, alocando-se socialmente apenas na vida domés- 
tica, privada, sendo assim, as relações familiares são a primeira das relações de opressão feminina, sobretudo pela normatividade social para o feminino e masculino, e no caso das mulheres a representação simbólica de maior valor, o de ser mãe.

Outros debates como os de Rosaldo e Atkinson (1975) tratam da "assimetria sexual" que se baseia na hierarquia entre o masculino e o feminino, elaborada pela cultura, resultando em dupla moral sexual: uma para homens (a liberdade sexual plena e até a necessidade de mostrar grande número de parcerias sexuais) e outra para mulheres, dentro do recato, da parcimônia sexual e a conexão entre a vida sexual com a maternidade. Estas autoras consideram que a libertação das mulheres estaria na recusa da maternidade, vislumbrando uma "cultura andrógina" e o desaparecimento do aprisionamento familiar e doméstico, na perspectiva da produção, do trabalho social.

Já para Rubin(1986) a opressão das mulheres estaria no sistema social que ao instaurar a divisão sexual do trabalho instituiria a dependência entre os sexos (mulheres dependendo dos homens como provedores) garantindo o casamento, o que obrigaria a formação da família. Para a autora é a família que ocupa um importante espaço de produção de desigualdades de gênero e de geração. Muitas vezes é em nome da família que a maior parte dos direitos das mulheres são desrespeitados.

Todas essas situações apontadas pelos estudos feministas - de casamento obrigatório e formação obrigatória da família, maternidade, centramento na vida doméstica com decorrente obstaculização ao trabalho social e a assimetria de sujeito sexual - e que são base para um menor poder das mulheres como sujeitos sociais e sujeitos de direitos, podem e devem ser pensadas também no seu sentido de vulnerabilidade à saúde.

O conceito de vulnerabilidade tem norteado a trajetória da epidemia, bem como a sua dinâmica. Ele nasceu na área dos Direitos Humanos, tendo sido incorporado ao campo da saúde a partir dos trabalhos realizados na Escola de Saúde Pública de Harvard por Jonathan Mann sobre a epidemia da Aids, nos anos de 1980.
Ampliado por Ayres, França, Calazans e Saletti (2003), no Brasil, o conceito de vulnerabilidade foi desdobrado em três situações concretas e particulares: individual, programática e social. Situam-se, nestes desdobramentos, as possibilidades de infecção e adoecimento como devidas às desigualdades sociais, iniquidades em saúde, inclusive de acesso a serviços e a práticas de prevenção, e a situações familiares e individuais de violação de direitos e de carências ou ausência de apoios para os comportamentos individuais, ampliando e complexificando as dimensões da vida de relações a serem consideradas na epidemia (Ayres, Calazans, \& França, 1998).

Ao deflagrar-se a aids como uma epidemia de doença infecciosa transmitida pelo contato direto entre pessoas, inicia-se uma série de formulações e julgamentos das condutas dessas pessoas, quando os primeiros diagnósticos surgem principalmente, frente ao fato da transmissão estar relacionada às relações sexuais e ao uso de drogas injetáveis. Ao caracterizar as populações que se haviam infectado nos anos de 1980 como populações masculinas que tinham práticas homo/bissexuais, ou seja, pelo fato da aids ter se concentrado entre os homens que faziam sexo com homens, vários estereótipos foram formulados, como os relacionados a uma doença incurável, contagiosa, "peste gay", com predominância de pessoas de classe social alta e dos grandes centros urbanos. O estigma então criado persiste ainda nos dias de hoje (Nascimento, Barbosa, \& Medrado, 2005).

Apesar de quase três décadas do surgimento da aids, e seu contínuo processo de transformação, nota-se através dos boletins epidemiológicos que a infecção pelo HIV aumenta em diferentes perfis populacionais.

Com o decorrer do tempo e com o avanço rápido da epidemia e a extrapolação da infecção relativamente aos chamados "grupos de risco"aqueles com comportamentos "inaceitáveis" - é que se percebeu a necessidade de novos enfoques e novas ações que repercutissem no controle da epidemia.

Há, hoje, um novo olhar epidemiológico, caracterizando a epidemia no Brasil; suas marcas são a pauperização, a juvenização, a interiorização e a feminização do HIV/Aids (Pinto, 
Pinheiro, Vieira, \& Alves, 2007). A década de 1990 foi marcada pelos avanços da terapia medicamentosa, pela heterossexualização do HIV e pelo controle da transmissão vertical (na gravidez). Ou seja, uma nova configuração da doença, até então relacionada a grupos específicos, agora extensivos às mulheres e bebês de mães soropositivas.

Portanto, a feminização exige importantes esforços que reduzam a vulnerabilidade das mulheres.

Diante dessas delimitações da vulnerabilidade, no caso das mulheres vivendo com HIV/ AIDS, as relações de gênero surgem como uma importante categoria articulada às vulnerabilidades individual, programática e social, o que se expressa na subordinação das mulheres ao sexo inseguro, às relações sexuais forçadas, a um modo de uso dos serviços em que essa subordinação e o comportamento de risco feminino não encontram nenhum apoio contra-cultural e valorização dos direitos das mulheres, e, por fim, se expressa individualmente na maior exposição à infecção e em diminuição do cuidado de si mesma.

Portanto, o conceito de vulnerabilidade valoriza os contextos relacionais que os indivíduos mantêm em sociedade, no trabalho, nos espaços públicos de circulação, nas famílias e nos serviços de saúde. Aspectos individuais nessas relações sociais permitem que no plano de cada indivíduo, sejam desenvolvidas relações mais protetoras ou ao contrário de maior exposição à infecção e ao adoecimento. Desse modo, cada indivíduo está em interação contextual, derivando das qualidades desses contextos a capacidade individual de proteção ou risco.

No caso das mulheres, essa vulnerabilidade pode ser trabalhada também dessa perspectiva cultural trazida pelo conceito de gênero, o que inclui entre as situações acima mencionadas relativas ao casamento, maternidade e comportamento sexual, a aceitação da violência no contexto familiar, em nome da formação e manutenção da família. Esse comportamento, que expressa uma vulnerabilidade específica, aponta importantes elementos para abordar a crescente presença das mulheres na epidemia.
Em estudo realizado por Santos et al. (2009), sobre contextos de vulnerabilidade para o HIV entre mulheres brasileiras, a autopercepção da infecção pelo HIV referida pelas mulheres entrevistadas, relacionava-se ao parceiro ter múltiplas parcerias sexuais ou ser bissexual. Este estudo com 3882 mulheres acima de 17 anos, sendo que 1777 eram portadoras do vírus HIV e 2045 sem diagnóstico conhecido para o HIV, também apontou que $32 \%$ das mulheres não vivendo com HIV/AIDS relataram que nunca usavam preservativos durante as relações sexuais e $46,1 \%$ o usavam às vezes. Tais porcentagens foram significativamente maiores para as mulheres vivendo com HIV/AIDS ao se referirem à época da infecção, $62,2 \%$ nunca usavam preservativos e $32,3 \%$ usavam às vezes. Outro dado apontado é que das mulheres vivendo com HIV/AIDS que tinham parceiro fixo com sorologia positiva $(n=509)$, $38,5 \%$ relataram fazer uso de preservativos de forma inconsistente (definida como nunca usar o preservativo durante as relações sexuais ou usar às vezes); esta proporção foi um pouco maior $(43,4 \%)$ para as mulheres com parceiro fixo com sorologia desconhecida $(n=193)$ e significativamente menor (20\%) quando o parceiro fixo tinha sorologia negativa $(n=396)$.

A questão da percepção da vulnerabilidade à exposição para o HIV se coloca quando as mulheres, deste estudo relataram que só conheceram o status sorológico em decorrência de adoecimentos, seja do companheiro, dela ou do filho. As demandas para a realização da sorologia ocorreram, por iniciativa própria, somente em $12 \%$ das mulheres e $30 \%$ por solicitação dos profissionais de saúde. Tal fato também já foi observado em outros estudos sobre a baixa percepção de risco das mulheres em relação ao HIV (Braga, Cardoso, \& Segurado, 2007; Filipe et al., 2000).

Implicada nessa baixa percepção de risco, estão as dificuldades em negociar o uso de preservativos, quando a proporção de mulheres infectadas pelos parceiros fixos e uso inconsistente de preservativos, foram referidas no estudo mencionado. Em outros estudos a situação de casamento como proteção para o HIV, a falta de informação sobre os modos de transmissão, ain- 
da são apresentados como importante vulnerabilidade das mulheres (Filipe et al., 2005).

O estudo também revelou que mulheres vivendo com HIV/Aids têm histórico significativo de situações de violência sexual: $22,1 \%$ entre as mulheres com HIV/Aids contra 13,2\% de mulheres que não vivem com HIV e aids.

Ampliando o leque das questões implicado na vulnerabilidade, a perspectiva de gênero como antes detalhada diversifica o modo como esse conceito é trabalhado na abordagem da epidemia de HIV/Aids, em que são consideradas as dimensões individual, programática e social.

Em estudo realizado com mulheres vivendo com HIV/Aids e que buscou as situações de violência experimentadas por essas mulheres (Lima, 2012), pudemos observar que a vulnerabilidade em todas as mencionadas dimensões é perpassada por roteiros de gênero tais como: a prática sexual como o dever da esposa; a banalização da violência de gênero pelo parceiro íntimo; as relações amorosas incondicionais e para sempre; a maternidade como a prioridade da mulher na sociedade; a família como valor dos valores para a boa qualidade de vida e para os cuidados. Tais referências permeiam a vida social das mulheres estudadas, criando situações em que as relações de gênero hegemônicas (vulnerabilidade de gênero no plano social), as concepções também hegemônicas de cuidar e relacionar-se com os serviços de saúde e as práticas profissionais (vulnerabilidade de gênero no plano programático) e as concepções de comportamentos individuais idealizados das mulheres, no adoecer e no cuidado de si mesma (vulnerabilidade de gênero no plano individual) constituem o efetivo comportamento cotidiano dessas mulheres. Isso aponta o 'dever de esposa', a 'banalização das violências', o ideal do 'amor incondicional', a 'maternidade para valorizar-se como sujeito' e a 'manutenção da família a qualquer custo', como questões de gênero a serem necessariamente abordadas com as mulheres, por exemplo em serviços de aconselhamento, para diminuir suas vulnerabilidades à epidemia.

Embora existam estudos que apontam a vulnerabilidade programática de mulheres para questões de gênero, entre elas a da violência contra as mulheres, as quais não são criticadas, mas reproduzidas no sentido hegemônico pelos profissionais de saúde (Schraiber, d'Oliveira, Falcão, \& Figueiredo, 2005), o fato é que mesmo serviços voltados à pessoas portadoras de HIV, não conseguiram modificar essa limitação que a normatividade de gênero impõe à organização dos serviços. Por exemplo, foi constatado, em pesquisa com usuárias de serviços de saúde em São Paulo, que mulheres que buscam a testagem para HIV, e/ou aquelas que são soropositivas relatam mais violência por parceiro íntimo em relação as mulheres que não passam/passaram por esta experiência (Barros et al., 2011). Mas esse resultado ainda não se traduz em mudanças na atenção às mulheres ou na inclusão da questão da violência como parte da abordagem das mulheres em geral pelos serviços de saúde.

Pautar estas temáticas da violência e da prevenção do HIV/Aids, no cotidiano dos serviços de saúde é necessário, para visibilizar e intervir nas situações de vulnerabilidade vivenciadas pelas mulheres às duas condições, que estão fortemente relacionadas às desigualdades nas relações de gênero. Trabalhar estas questões no contexto da assistência nos serviços de saúde, especialmente da atenção primária, é fundamental para pensar a saúde de mulheres como sujeitos de direitos. As mulheres em situação de violência buscam os serviços de saúde com frequência, porém não há resolutividade para suas demandas, pela invisibilidade conferida às situações de violência (Schraiber et al., 2007). Em relação ao HIV/Aids, a testagem sorológica é realizada não só nos serviços especializados, mas na atenção primária, principalmente no atendimento ao pré-natal, momento este nem sempre acompanhado de aconselhamento (Praça $\&$ Barrancos, 2007). O aconselhamento é uma das atividades que valoriza o processo de escuta, de acolhimento das dúvidas, principalmente em relação às implicações do teste na vida das pessoas, e de seus parceiros, que buscam por atendimento. É neste espaço de escuta, que o profissional deve oferecer alternativas terapêuticas, como também, proporcionar e facilitar vínculos para que o sujeito sinta-se acolhido para relatar suas necessidades. Apesar dos avanços ocorridos ao 
longo dos anos no atendimento especializado em aids, a revelação do diagnóstico é sempre uma situação difícil, em função da responsabilidade e consequências psicológicas, sociais e éticas que o resultado pode trazer para o indivíduo.

Destacamos, também, que o Ministério da Saúde tem se preocupado com o tema da violência contra as mulheres e produzido documentos, norteadores da assistência no país, porém os protocolos estabelecidos para a assistência às mulheres vítimas de violência doméstica nem sempre são implantados, assim como nem sempre é realizada a abordagem da intersecção já estudado em pesquisas entre a vulnerabilidade às DST/HIV/Aids e a violência conjugal. Assim, a própria política proposta tem tido poucas repercussões significativas para o enfrentamento desta discussão no âmbito programático.

Portanto há obstáculos para o cuidado integral aos sujeitos nos serviços de saúde, no caso às mulheres vítimas de violência doméstica. $\mathrm{O}$ reconhecimento da interdependência das situações de vulnerabilidade a que estão expostas, pode contribuir para analisar as estratégias de prevenção da infecção, ao considerar as dimensões mencionadas acima.

Assim como os casos de violência estão nos serviços de saúde, muitas vezes pouco reconhecidos, como aponta d'Oliveira, Schraiber, Hanada e Durand (2009), estratégias para abordar o tema violência reposiciona as necessidades de repensar os atendimentos na área do aconselhamento. A falta de orientações específicas nos documentos norteadores de como abordar a prevenção às DST/HIV/AIDS junto das mulheres em situação de violência, muitas vezes crônica, perpetrada por parceiros íntimos, se constitui numa importante falha dos documentos normativos, justamente porque esta é uma das maiores vulnerabilidade das mulheres.

Mas é preciso também reconhecer que abordar o tema violência é uma questão bastante difícil que exige cuidados éticos e técnicos especiais, como a garantia da relação moralmente isenta, da construção de vínculos de confiança, da privacidade, do sigilo, do acolhimento do relato e da mulher independentemente da história narrada, assim como da decisão compartilhada em termos de uma proposta assistencial que via de regra representa também o acionamento de rede intersetorial existente, garantindo os princípios de não julgamento e respeito às decisões da mulher (d'Oliveira et al., 2009). A violência, afinal, não é apenas mais um dos temas das desigualdades de gênero, embora seja isso também. A violência é um tema que, pertencendo às questões de gênero, radicaliza a desigualdade de poder das relações entre mulheres e homens, por representar a mulher não como um sujeito subordinado, mas como um não sujeito. Como mostra a literatura acerca da violência de gênero contra as mulheres (Schraiber et al., 2005), nas situações de violência, os sujeitos que a sofrem, no caso as mulheres, restam anulados como tal na relação, silenciados ou reduzidos a objetos das agressões ou abusos.

Pode-se ampliar essas exigências no trato de um tema tão complexo quanto a violência se esta ocorre com mulheres portadoras do HIV, pois além do que já se mencionou, há que se agregar os requisitos da postura profissional específica à assistência às pessoas que vivem com o HIV. Estas não divergem daquelas relativas à assistência a vítimas de violência, mas somam, ampliando as exigências de cuidados éticos e técnicos apropriados.

Cabe, desse modo, olhar de modo mais atento à própria questão da violência já para as mulheres que vivem com HIV/Aids, examinando de que modo uma e outra condição potencializam e agravam as vulnerabilidade de gênero dessas mulheres.

\section{A Violência no Repertório das Vulnerabilidades das Mulheres}

Diversos estudos têm destacado a importância que o viver cotidiano em um contexto de violência assume para as mulheres portadoras do HIV/aids. A violência, em especial aquela perpetrada pelo parceiro íntimo, alimenta muito as questões problemáticas de cuidar de sua saúde e, ao mesmo tempo, desempenhar-se como esposa ou companheira e mãe. Esse destaque aqui dado também deriva do fato de que a violência e os estudos acerca dela tornaram mais evidentes as questões de gênero para a própria epidemia do HIV/Aids. Além disso, nossa pesquisa com mulheres portadoras do HIV, mostra que a violência 
é um marco no reconhecimento da condição de portadora de uma doença que, além de transmissível, é crônica e estigmatizante (Lima, 2012).

A hipótese de a feminização estar alimentada pelo crescimento da violência contra os corpos, mentes, saúde e direitos de mulheres e meninas no mundo todo, tem sido considerada em vários estudos (Costa, 2000). Em 2002, a Organização Panamericana de Saúde, órgão da Organização Mundial de Saúde (OMS), mencionava em seu relatório que a violência sexual e doméstica e o HIV/Aids são dois dos mais graves problemas de saúde e de desenvolvimento humano na America Latina e Caribe (OMS, 2003). Os que estudam a violência por parceiro íntimo no Brasil, por exemplo, têm apontado para tal situação como responsável por maiores taxas de infecção pelo HIV (Barros et al., 2011), e pelo aumento da epidemia em casais, seja de formação hetero ou homossexual (Schraiber et al., 2007), corroborando diversos estudos epidemiológicos internacionais .

O sexo por obrigação marital, sem consentimento ou por medo, acarreta maior vulnerabilidade à infecção por doenças sexualmente transmissíveis, devido a falta de proteção e as possíveis lesões genitais ou anais que podem favorecer a infecção (Choi, Binson, Adelson, \& Catania, 1998; Decker et al., 2009; Jewkes, 2002).

Aids e violência se assemelham por associar-se à violação ou negligência de direitos humanos, às iniqüidades como as de gênero, de classe social ou de raça. Ambos são resultados de cenários culturais complexos que produzem atitudes e práticas cotidianas, além disso, parecem reforçarem-se mutuamente. Assemelham-se, também, nas relações provocadas pelos conflitos familiares em razão de uma doença que remete a preconceitos e desqualificação da mulher por ser portadora do HIV. Nesse caso, a violência pode decorrer do diagnóstico da infecção (Schraiber, Barros, \& Castilho, 2010).

A revelação do diagnóstico ao parceiro, principalmente se o diagnóstico dele for negativo, poderá desencadear violência (Maman et al., 2002). Outra questão que se coloca é a de que mulheres que vivem situações de violência podem ter dificuldades na adesão ao tratamento com repercussões importantes no cuidado de sua saúde. Isto porque, mesmo quando o parceiro foi anteriormente diagnosticado ou vem a saber de sua condição pela testagem realizada por sua parceira, o fato de a mulher ser também soropositiva ampliam as dificuldades para o sexo seguro, surgindo novas formas de recusa no uso dos preservativos, pois estando ambos infectados, não seria mais necessária essa prevenção. Além disso, quando a mulher se infecta, ela saí do lugar social da cuidadora mais apta para também estar na condição de necessidade de cuidados, alterando sua condição sócio-culturalmente valorizada, de mulher, nas referências tradicionais de gênero (Barros et al., 2011).

Tendo em vista que a própria violência contra as mulheres não foi alvo de pesquisa sistemática, e em especial no campo da Saúde, a não ser a partir da segunda metade dos anos 1990 (Schraiber, D'Oliveira, \& Couto, 2006), ela não foi considerada até recentemente como uma questão quer para o diagnóstico, quer, sobretudo, implicada no tratamento de mulheres portadoras do HIV.

Há ainda, na política que rege a atenção a vitimas de violências até certo ponto um paradoxo, pois no caso da violência sexual por estranhos, medidas preventivas são disponibilizadas nos serviços de saúde para as mulheres, e neste caso a quimioprofilaxia do HIV será ofertada nos serviços de saúde. Mas quer se trate de violência doméstica, que vulnerabiliza a mulher de modo mais crônico e reiterado, quer se trate de mulher já portadora do HIV, os serviços já não dispõe de programas apropriados de intervenção ou de prevenção das infecções secundárias pelo próprio HIV.

No contexto do HIV/Aids, a UNAIDS (2008) refere em seus relatórios que vários estudos no mundo confirmam a relação entre violência contra as mulheres e a infecção pelo HIV. Revela que uma em cada três mulheres no mundo já foi espancada, forçada ao sexo ou abuso, e geralmente por algum conhecido (UNAIDS, 2008). Tais referências indicam que as mulheres portadoras do HIV tiveram maior probabilidade de ter vivido situações de violência, sendo estas sobretudo por seus parceiros mais do que por estranhos, e as que sofrem violência teriam maior 
propensão à infecção e a outros adoecimentos, especialmente os relacionados à saúde mental e emocional.

Reforçado pelo estudo de Mello, Segurado e Malbegier, (2010), questões de ordem emocional, como tristeza e depressão têm maior prevalência em pessoas soropositivas para o HIV, cerca de 1,99 mais elevada quando comparada com pessoas soronegativo (Ciesla \& Roberts, 2001). As mulheres são as mais atingidas, sobretudo pelas dificuldades relacionadas à vergonha, estigma, ansiedade, além da sobrecarga que na condição de mãe e cuidadora se sobrepõe ao cuidado de si. Neste sentido o estudo aponta que mulheres com carga viral alta são as que apresentaram maior quadro depressivo. Assim, como o estresse e a depressão são sintomas associados a uma diminuição da imunidade, implicando no controle da replicação do vírus, fatores associados a depressão em mulheres vivendo com HIV/ Aids também estão relacionados à falta de apoio emocional, questões financeiras, moradia, pobreza e baixa escolaridade.

A violência é um fator que agrava tal situação, uma vez que estudos mostram a grande associação entre sofrer violência e transtornos mentais e mesmo ideação e tentativas de suicídio (Devries et al., 2011; Ellsberg, Jansen, Heise, Watts, \& Garcia-Moreno, 2008). A experiência de situações de violência, portanto, representa destacado aspecto da vulnerabilidade das mulheres já que vem somar preocupações para com a revelação de seus diagnósticos para os parceiros e para familiares; preocupações quanto ao modo como se infectaram e a prevenção das infecções secundárias.

A manutenção de situações violentas e as dificuldades em lidar com tais preocupações, durante o processo de tratamento, podem contribuir com os adoecimentos secundários à soropositividade e com a falta do cuidado. Schraiber et al. (2003) destaca que a baixa autoestima derivada das situações de violência, leva a modos de relação com a saúde e o uso de serviços que resultam em negligência de cuidados de si própria ou de outros, tais como na entrada tardia para a atenção pré-natal ou a manutenção dos cuidados preventivos relativamente às suas crianças.
A literatura também reforça a necessidade de intervenções nos serviços de saúde, com abordagens qualificadas para o cuidado de tão importante questão de saúde pública. O tema tem sido estudado como uma das invisibilidades da violência como questão social e de saúde (Heise et al., 1999; Heise \& Garcia-Moreno, 2002). No Brasil, foi o alvo primeiro das políticas públicas voltadas à criação das delegacias especiais de polícia voltadas para os direitos das mulheres (DDM e DEAM), também se verifica a tendência dos profissionais em considerar que se trata de questões relacionadas à justiça e que pouco podem contribuir para o evento (Schraiber et al., 2005). Neste sentido, a violência cometida por parceiro íntimo é considerada um problema privado em que os profissionais de saúde não se sentem preparados a atuar, e os serviços pouco estruturados para a condução de queixas que não possam ser referidas em um modelo medicalizado.

Kiss e Schraiber (2011) argumentam que a invisibilidade da violência, no campo da saúde, ocorre pela "recusa tecnológica", isto é, quando os profissionais evitam abordar essa situação em sua atuação assistencial por considerarem que não existem tecnologias competentes que possam ser trabalhadas no âmbito dos serviços de saúde.

Parte dessas representações se reforça pelo caráter bastante íntimo dessas situações de violência. De fato os estudos têm chamado a atenção para este caráter e as dificuldades de revelação por parte das mulheres ou da indagação por parte dos profissionais nos serviços (Kiss \& Schraiber, 2011; Schraiber et al., 2003).

Muitas vezes a violência de gênero está situada no espaço das relações amorosas ou de intimidade, o que permite considerar as representações e concepções sobre o amor nas relações de intimidade e conjugalidade, além das expectativas normativas para a maternidade, componentes de situações de maior vulnerabilidade para as mulheres relativamente à infecção pelo HIV. Neste sentido as expectativas conjugais e familiares, com base no referencial de gênero, também se aliam à permanência das mulheres em situações de violência por muitos anos, mes- 
mo as de agressões graves e sob a alegação das expectativas amorosas e de resgate de relações conjugais e de intimidade idealizadas (Neves, 2008).

\section{Concepções Amorosas e Conjugalidade: A Vulnerabilidade das Mulheres nas Relações de Intimidade e Sexuais}

Na construção social da normatividade para ser homem e mulher, o lugar simbólico da família e das parcerias amorosas organiza crenças e códigos de valores que podem favorecer a exposição das mulheres a situações de risco e produzir impactos na epidemia da aids. Um desses códigos diz respeito, como dito, à aceitação de uma dupla moral sexual, a do comportamento de homens e a do comportamento de mulheres e que, no caso das mulheres, pode torná-las mais vulneráveis ao HIV, por dificultar tanto o reconhecimento de que estejam em situações de risco, quanto nas decisões e no exercício de comportamentos sexuais mais seguros(Bastos \& Sczwarcwald, 2000).

Diante disto o tema da sexualidade ganha destaque no campo da saúde pública por ser a via mais importante da infecção, e a prevenção com o uso de preservativos ser recomendada. No entanto, isso implica em explicitar fatos da intimidade, do considerado proibido, do escondido e da moral. Embora a partir do século XVIII, a sexualidade ganhe importância dentro do casamento, ainda não é tema tratado tão abertamente. Se até o século XVIII a escolha amorosa e pela paixão (dos homens) era vivenciada dentro das relações de adultério e a sexualidade conjugal vista como uma função especificamente para a reprodução e não para o prazer, a noção de intimidade na contemporaneidade constitui possibilidade de aglutinar elementos como o amor e o afeto, a valorização pessoal, o cuidado, a interdependência, a mutualidade, a confiança e o comprometimento (Araujo, 2002) e permite distinções entre vida amorosa íntima da mulher e sua vida amorosa como mãe na família. Com essa forma de abordagem teríamos melhor aproximação das novas situações da mulher na família, assim como melhor compreensão, para o caso das mulheres portadoras do HIV, o quanto esta condição de saúde altera, intensificando vul- nerabilidades, quer no desempenho de mulher em relações de intimidade, em especial ao lidar com sua sexualidade, quer como mãe e cuidadora da família. Isto porque, a despeito das novas posições objetivas da mulher na família, como trabalhadora social e também provedora da família, por exemplo, observamos em nossa pesquisa com mulheres portadoras de HIV (Lima, 2012) que essas mudanças na posição da mulher como trabalhadora social não necessariamente transformam também as representações mais tradicionais de gênero quanto ao papel da mulher como cuidadora e como mãe.

Concepções idealizadas de amor também surgem como um argumento fundamental, mas ao mesmo tempo castrador da autonomia das mulheres, quando elas são as portadoras do HIV e seus parceiros, não. Outro aspecto a ser lembrado é que nas situações opostas, em que os homens são os portadores e as mulheres não, o amor, que se expressa pelo cuidar e permanecer em relação conjugal torna-se uma espécie de obrigação da mulher, ao menos ela assim o identifica quanto à permanência de seu envolvimento com seu parceiro, zelando pelo tratamento e a saúde dele.

\section{Considerações Finais}

Dois grandes aspectos das questões de gênero foram aqui abordados no sentido de uma releitura da epidemia do HIV/aids entre as mulheres que permita diminuir sua vulnerabilidade, inspirando também novas práticas de saúde: as relações de intimidade (afetivo-sexuais e amorosas) e as relações de violência. Ambas, de modo diverso, potencializam as vulnerabilidades social, programática e individual das mulheres, enquanto vulnerabilidades de gênero, o que deve considerado tanto em estudos científicos para a compreensão da condição feminina no contexto da epidemia de Aids, quanto nas políticas de atenção integral às mulheres nos serviços de saúde.

As vulnerabilidades de gênero expressamse na subordinação das mulheres ao sexo inseguro, às relações sexuais forçadas, à renúncia de exercício da própria sexualidade de modo mais satisfatório, a um temor de não responder pela 
maternidade ou mesmo pelo cuidado dos filhos e do parceiro. Também se expressam no modo de uso dos serviços, em que essa subordinação de gênero e o comportamento de risco feminino não encontram nenhum apoio com valorização dos direitos das mulheres. Por outro lado, nessa mesma relação programática com os serviços, expressam-se como uma ausência de discriminação positiva, com cuidados especiais, para as dificuldades adicionais da mulher soropositiva ante ao exercício da maternidade esperado assim como até mesmo ante a expectativa de vir a ser mãe. Por fim, na vulnerabilidade individual, a de gênero se expressa na maior exposição à infecção e em diminuição do cuidado de si mesma.

\section{Referências}

Araujo, M. F. (2002). Amor, casamento e sexualidade: Velhas e novas configurações. Psicologia: Ciência e Profissão, 22(2), 70-77.

Ayres, J. R., Calazans, G. J., \& França, I., Jr. (1998). Vulnerabilidade do adolescente ao HIV/Aids. In E. Vieira (Org.), Seminário gravidez na adolescência (pp. 97-109). Rio de Janeiro, RJ: Associação Saúde da Família.

Ayres, J. R., França, I., Jr., Calazans, G. J., \& Saletti, H. C., Filho. (2003). O conceito de vulnerabilidade e as práticas de saúde: Novas perspectivas e desafios. In D. Czeresnia \& C. M. Freitas (Orgs.), Promoção da saúde: Conceitos, reflexões, tendências (pp. 117-139). Rio de Janeiro, RJ: Editora da Fundação Oswaldo Cruz.

Barros, C. R. S., Schraiber, L. B., \& França, I., Jr. (2011). Associação entre violência por parceiro íntimo contra a mulher e infecção por HIV. Revista de Saúde Pública, 45, 365-372.

Bastos, F. I., \& Szwarcwald, C. L. (2000). AIDS e pauperização: Principais conceitos e evidências empíricas. Cadernos de Saúde Pública, 16(1), 65-76.

Braga, P., Cardoso, M. R. A., \& Segurado, A. A. C. (2007). Gender differences in survival in an HIV/AIDS cohort from São Paulo, Brazil. AIDS Patient Care and STDs, 21, 321-328.

Choi, K. H., Binson, D., Adelson, M., \& Catania, J. A. (1998). Sexual harassment, sexual coercion, and HIV risk among U.S. adults 1849 years. AIDS and Behavior, 2(1), 33-40. doi:10.1023/A:1022355206905.
Ciesla, J. A., \& Roberts, J. E. (2001). Meta-analysis of the relationship between HIV infection and risk for depressive disorders. American Journal of Psychiatry, 158(5), 725-730.

Costa, S. M. S. (2000). Vivendo com aids e enfrentando a violência: A experiência das adolescentes (Dissertação de mestrado, Escola Nacional de Saúde Pública, Fundação Oswaldo Cruz, Rio de Janeiro, RJ, Brasil).

Decker, M. R., Seage, G. R., Hemenway, D., Raj, A., Saggurti, N., \& Balaiah, D. (2009). Intimate partner violence functions as both a marker and risk factor for women's HIV infection: Findings from Indian husband-wife dyads. Journal of Acquired Immune Deficiency Syndromes, 51(5), 593-600. doi: 10.1097/QAI.0b013e3181a255d6

Devries, K., Watts, C. H., Yoshihama, M., Kiss, L. B, Schraiber, L. B., Deyessa, N., ...Garcia-Moreno, C. (2011). Violence against women is strongly associated with suicide attempts: Evidence from the WHO multi-country study on women's health and domestic violence against women. Social Science \& Medicine, 73(1), 79-86.

d'Oliveira, A. F. P. L., Schraiber, L. B., Hanada, H., \& Durand, J. (2009). Atenção integral à saúde de mulheres em situação de violência de gênero: Uma alternativa para a atenção primária em saúde. Ciência: Saúde Coletiva, 14(4), 10371050. Recuperado em http://dx.doi.org/10.1590/ S1413-81232009000400011

Ellsberg, M., Jansen, H. A. F. M., Heise, L., Watts, C. H., \& Garcia-Moreno, C. (2008). Intimate partner violence and women's physical and mental health in the WHO multi-country study on women's health and domestic violence: An observational study. Lancet, 371, 1165-1172.

Filipe, E. M. V., Batistella, E., Pine, A., Santos, N. J. S, Nascimento, A. M. G., Barbosa, C. S., \& Medrado, B. (2005). Mulheres em Camaragibe: Representação social sobre a vulnerabilidade feminina em tempos de AIDS. Revista Brasileira de Saúde Materno Infantil, 5(1), 77-86.

Filipe, E. M. V, Bugamelli, L., Leme, B., Santos, N. J. S., Garcia, S., \& Paiva, V. (2000). Risk perception and counselling among HIV-positive women in São Paulo, Brazil. International Journal of STD \& Aids, 11, 112-114.

Firestone, S. (1976). A dialética do sexo. Rio de Janeiro, RJ: Labor Brasil.

Franchetto, B., Cavalcanti, M. L., \& Heilborn, M. L. (1981). Antropologia e feminismo: Vol. 1. Perspectivas antropológicas da mulher. Rio de Janeiro, RJ: Zahar. 
Heise, L., Ellsberg, M., \& Gottemoeller, M. (1999). Ending violence against women. Population Reports, 27(4), 1-43.

Heise, L., \& Garcia-Moreno, C. (2002). Intimate partner violence. In E. G. Krug, L. L. Dahlberg, J. A. Mercy, A. B. Zwi, \& R. Lozano (Eds.), World Report on violence and health (pp. 91-121). Geneva, Switzerland: World Health Organization.

Huebner, D. M., Rebchook, G. M., \& Kegeles, S. (2004). Experiences of harassement, discrimination, and physical violence among young gay and bisexual men. American Journal of Public Health, 94(7), 1200-1203.

Jewkes, R. (2002). Intimate partner violence: Causes and prevention. Lancet, 359(9315), 1423-1429. doi: 10.1016/S0140-6736(02)08357-5

Joint United Nations Program on HIV/AIDS. (2008). Report on the global AIDS epidemic: Executive summary. Geneva, Switzerland: Author.

Kiss, L. B., \& Schraiber, L. B. (2011). Temas médico-sociais e a intervenção em saúde: A violência contra mulheres no discurso dos profissionais. Ciência: Saúde Coletiva, 16(3), 1943-1952.

Lima, M. (2012). Vulnerabilidade de gênero e mulheres vivendo com HIV e Aids: Repercussões para a saúde (Tese de doutorado, Faculdade de Medicina, Universidade de São Paulo, SP, Brasil).

Maman, S., Mbwambo, L. K., Hogan, N. M., Kilonzo, G. P., Campbell, J. C., \& Weiss, E. (2002). HIV-positive women report more lifetime partner violence: Findings from a voluntary counseling and testing clinic in Dar es Salaam, Tanzania. American Journal of Public Health, 92(8), 1331-1337.

Mello, V. A., Segurado, A. A. C., \& Malbegier, A. (2010). Depression in women living with HIV: Clinical and psychosocial correlates. Archives of Women's Mental Health, 13, 193-199.

Ministério da Saúde. (2011). Plano Integrado de enfrentamento à feminização da aids e outras DST. Recuperado em http://www.aids.gov.br

Nascimento, A. M. G., Barbosa, C. S., \& Medrado, B. (2005). Mulheres em Camaragibe: Representação social sobre a vulnerabilidade feminina em tempos de aids. Revista Brasileira de Saúde Materno Infantil, 5(1), 77-86.

Neves, A. S. (2008). Amor, poder e violências na intimidade. Os caminhos entrecruzados do pessoal e do politico. Coimbra, Portugal: Quarteto.
Organização Mundial da Saúde. (2003). Relatório Mundial sobre violência e Saúde. Geneva, Switzerland: Organização Pan-Americana da Saúde.

Pinto, A. C. S., Pinheiro, P. N. C., Vieira, N. F. C., \& Alves, M. D. (2007). Compreensão da pandemia da Aids nos últimos 25 anos. Jornal Brasileiro de Doenças Sexualmente Transmissíveis, 19(1), 45-50.

Praça, N. S., \& Barrancos, J. T. G. (2007). Teste anti-HIV e aconselhamento no pré-natal: Percepção de puérperas [HIV screening and counseling in antenatal care: Perception of postpartum women]. Revista Gaúcha de Enfermagem, 28(1), 106-116.

Reed, E. (1973). Problems of women liberation. A Marxist approach. New York: Parthfinder Press.

Rosaldo, M. Z., \& Atkinson, J. (1975). Man the hunter: Metaphors for the sexes in ilongot magical spells. In R. Willis (Ed.), The interpretation of symbolism (pp. 43-75). London: Malaby.

Rubin, G. A. (1986). Circulação de mulheres: Notas sobre a 'economia política' do sexo. Nueva Antropologia, 8(30), 95-145.

Santos, N. J. S., Barbosa, R. M., Pinho, A. A., Villela, W. V., Aidar, T., \& Filipe, E. M. V. (2009). Contextos de vulnerabilidade para o HIV entre mulheres brasileiras. Cadernos de Saúde Pública, 25(Supl. 2), s321-s333. Recuperado em http:// dx.doi.org/10.1590/S0102-311X2009001400014

Schraiber, L. B., Barros, C. R. S., \& Castilho, E. A. (2010). Violência contra as mulheres por parceiros íntimos: Usos de serviços de saúde. Revista Brasileira de Epidemiologia, 13(2), 237-245.

Schraiber, L. B., d’Oliveira, A. F. P., Falcão, M. T. C., Figueiredo, W. S. (2005). Violência dói e não é direito. A violência contra a mulher, a saúde e os direitos humanos. São Paulo, SP: Editora da Universidade Estadual Paulista Júlio de Mesquita Filho.

Schraiber, L. B., d'Oliveira, A. F., Hanada, H., Figueiredo, W., Couto, M., Kiss, L., ...Pinho, A. (2003). Violência vivida: A dor que não tem nome. Interface - Comunicação, Saúde, Educação, 7(12), 41-54.

Schraiber, L. B., d'Oliveira, A. F. P. L., França, I., Jr., Diniz, S., Portella, A. P., Ludermir, A. B., ...Couto, M. T. (2007). Prevalência da violência contra a mulher por parceiro íntimo em regiões do Brasil. Revista de Saúde Pública, 41(5), 797-807. 
Schraiber, L. B., D’Oliveira, D. F., \& Couto, M. T. (2006). Violência e Saúde: Estudos científicos recentes [Edição especial]. Revista de Saúde Pública, 40, 112-20.

Scott, J. W. (1990). Gênero: Uma categoria útil de análise histórica. Educação e Realidade (Porto Alegre), 16(2), 5-22.

Teitelman, A. M., Ratclitte, S. J., Dichter, M. E., \& Sullivan, C. M. (2008). Recent and past intimate partner abuse and HIV risk among young women. Journal of Obstetric, Gynecologic, and Neonatal Nursing, 37, 219-227.
Wong, C. F., Weiss, G., Ayala, G., \& Kiple, M. D. (2010). Discrimination, violence and illicit drug use among young men who have sex with men. AIDS Education and Prevention, 22(4), 286298.

Recebido: 05/09/2012

$1^{a}$ revisão: 30/10/2012

Aceite final: 30/11/2012 